\title{
MODELS FOR ANOMALIES IN CONDENSED MATTER DEUTERIDES
}

\author{
PETER L. HAGELSTEIN \\ Research Laboratory of Electronics, \\ Massachusetts Institute of Technology, \\ Cambridge, MA 02139 ,USA \\ and \\ Spindletop Corporation, Mountain View, CA 94040 \\ E-mail: plh@mit.edu
}

\begin{abstract}
Models based on phonon exchange for excess heat production in Fleischmann-Pons experiments are considered. In the case that sufficient phonon exchange occurs to stabilize intermediate states containing a neutral, then a model in which excitation is transferred from the $\mathrm{D}_{2} /{ }^{4} \mathrm{He}$ system to a strongly coupled quantum system made up of an oscillator (highly-excited phonon mode) and a Dicke system (ground state and excited state receiver nuclei) seem appropriate. We find that a coupled Dicke system and oscillator can support energy coupling in the case of strong coupling. We present evolution equations for resonant coupled Dicke systems, augmented with loss. An update is provided on phonon exchange in nuclear calculations.
\end{abstract}

\section{Introduction}

In 1989 there were announcements made of observations of anomalies in metal deuterides: Fleischmann and Pons presented observations of an excess heat effect in PdD [1] and Jones presented observations of low-level neutron emission in TiD [2]. Since then, considerable experimental and theoretical work has been reported on these and on other anomalies. As has been noted often over the years, the existence of any such effects would require a significant revision of nuclear physics to explain. We have focused our efforts on understanding the implications of these new experiments on theory, as well as on the development of models that may be relevant [3-5].

In the nuclear physics literature, the predominant view is that nuclear fusion reactions that occur in condensed matter can be understood from idealized models in which the local environment is replaced by vacuum. The basic argument is that in order to react nuclei must approach to within a few fermis, and that the resulting reaction is completed with fast-moving well-separated product nuclei long before information concerning the reaction can reach neighboring atoms on an atomic scale.

Such a picture cannot be relevant to the excess heat effect in Fleischmann-Pons type experiments, in which a large amount of energy (apparently of nuclear origin) 


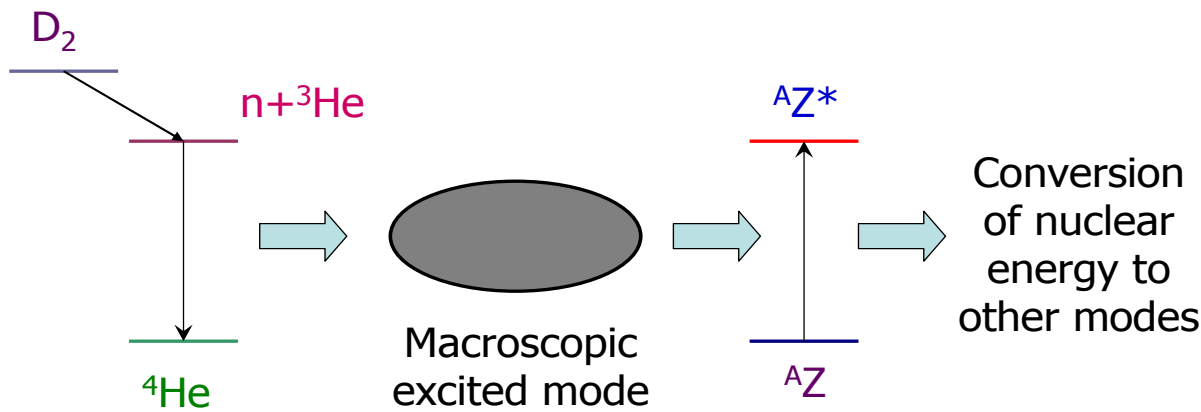

Figure 1. Scheme proposed for excess energy production. Molecular deuterium in condensed matter makes transitions to ${ }^{4} \mathrm{He}$ states through intermediate $\mathrm{n}+{ }^{3} \mathrm{He}$ states, with stabilization of the intermediate state through large angular momentum transfer associated with phonon exchange. The excitation is transferred to other species, which undergo fast phonon-mediated excitation transfer reactions among themselves, transferring energy to the excited phonon mode a few tens of phonons at a time.

appears and commensurate ${ }^{4} \mathrm{He}$ is detected. The experimental results are consistent with some new kind of reaction mechanism that acts as if

$$
\mathrm{d}+\mathrm{d} \longrightarrow{ }^{4} \mathrm{He}+23.8 \mathrm{MeV} \text { (heat) }
$$

In vacuum, two deuterons can react to produce ${ }^{4} \mathrm{He}$; however, energy and momentum conservation results in the energy being carried off by an energetic gamma. No such gammas or other energetic particles are present in association with the Fleischmann-Pons excess heat effect. In our view this indicates that the lattice participates in the reaction in some way, and that vacuum models will not be relevant. Our approach then is based on including the lattice (or more generally, the condensed matter environment) in the initial formulation at the outset [3-5]. In such a formulation one finds that phonon exchange can occur during the fusion process. For fast incoherent fusion reactions, the exchange of a phonon or two does not impact the fusion rate or products in a significant way, so that the predictions of vacuum models are generally preserved within the new formulation. The new model opens the door to a coupling between nuclear reactions that occur at different sites, as long as two or more phonons are exchanged at each site with a highly excited common phonon mode. In this case, there is the possibility of new second-order or higher-order quantum processes. We have discussed these ideas in some detail in our earlier ICCF conference papers, to which the interested reader is referred.

\section{Model for excess heat production}

At present, we are considering a model for excess heat production that is illustrated schematically in Figure 1. There are a number of ideas that are incorporated in this scheme. All transitions are mediated by phonon exchange (or by the exchange 
of similar quanta of a macroscopic condensed matter system that produces local acceleration of nuclei). In order to couple to the lattice, all individual nuclear transitions must involve a neutral, such as $\mathrm{n}+{ }^{3} \mathrm{He} \rightarrow{ }^{4} \mathrm{He}$, so that the initial and final states appear to the lattice to have different mass (since the lattice does not see neutrals). In the case of ${ }^{A} \mathrm{Z}$, the excited state $\left({ }^{A} \mathrm{Z}\right)^{*}$ is one in which there is a neutron or neutron cluster and a lower mass daughter with charge Z. Excitation is transferred from the $\mathrm{D}_{2} /{ }^{4} \mathrm{He}$ system on the right to the receiver nuclei on the left. In our early work we considered that ${ }^{4} \mathrm{He}$ would be appropriate receiver nuclei in this sense, but it is clear that other nuclei such as Pd or other host lattice nuclei should be able to participate, with neutron-rich isotopes being able to couple more easily to the lattice. As in our earlier publications, we consider the initial fuel to consist of molecular $\mathrm{D}_{2}$ in condensed matter (we note that a similar scheme should be possible for $\mathrm{HD}$ ).

To analyze this kind of scheme, we require two different basic tools. On the one hand, we need to analyze the microscopic interactions between nucleons in order to understand phonon exchange and develop estimates for transition matrix elements. In addition, we would like to understand the structure of the intermediate $n+{ }^{3} \mathrm{He}$ state, and the stability of excited neutral plus daughter states in general that are involved in the fast excitation transfer on the receiver side. On the other hand, we need models that are relevant to the many-site version of the problem. For example, we have discussed in previous papers that such models give rise to Dicke enhancement factors, and that tunneling in the coherent version of the problem appears through a rate linearly dependent on the Gamow factor, rather than through the square of the Gamow factor as in the case of incoherent transitions. In what follows, we will consider both problems.

\section{Coupled oscillator and two-level systems}

To address many site issues, we have been investigating models made up of two-level systems and an oscillator. The simplest example of a model that implements the ideas outlined above is given by

$$
\begin{aligned}
\hat{H}=\frac{\Delta E}{2} & \hat{\Sigma}_{z}^{(1)}+\frac{\Delta E}{2} \hat{\Sigma}_{z}^{(2)}+\hbar \omega_{0} \hat{a}^{\dagger} \hat{a} \\
& +V e^{-G}\left[\hat{\Sigma}_{+}^{(1)}+\hat{\Sigma}_{-}^{(1)}\right]\left(\hat{a}+\hat{a}^{\dagger}\right)^{m_{1}}+U\left[\hat{\Sigma}_{+}^{(2)}+\hat{\Sigma}_{-}^{(2)}\right]\left(\hat{a}+\hat{a}^{\dagger}\right)^{m_{2}}
\end{aligned}
$$

In this model the $\mathrm{D}_{2} /{ }^{4} \mathrm{He}$ system is represented by the first set of two-level systems, under the assumption that sufficient phonon exchange is present to allow transitions through the $\mathrm{n}+{ }^{3} \mathrm{He}$ intermediate state with negligible loss. The $\mathrm{D}_{2}$ and ${ }^{4} \mathrm{He}$ states are modeled as equivalent two-level systems (superscript 1 here), with transitions between these states represented with a hindered (by a tunneling factor $e^{-G}$ ) transition involving the exchange of up to $m_{1}$ phonons. The receiver side nuclear states 


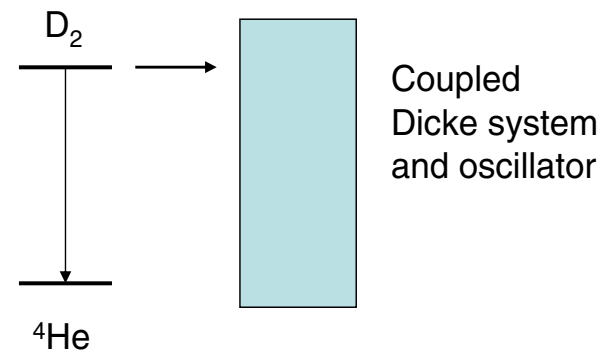

Figure 2. In the limit that the receiver-side two-level systems are strongly coupled to the oscillator, then it is useful to think in terms of two-level systems coupled to a coupled oscillator and Dicke system hybrid.

are also modeled as equivalent two-level systems (superscript 2) with unhindered transitions involving the exchange of up to $m_{2}$ phonons.

\subsection{Receiver as coupled two-level system and oscillator}

In this model, the receiver-side system alone is composed of two-level systems that is strongly coupled to an oscillator. Since transitions involving the first set of twolevel systems are weak, we can think of the system in terms of weak transitions from two-level systems (the $\mathrm{D}_{2} /{ }^{4} \mathrm{He}$ systems) to a hybrid coupled oscillator and Dicke system. This is picture schematically in Figure 2.

If we adopt such a view, then we become interested in understanding the hybrid system. The receiver-side Hamiltonian is

$$
\hat{H}_{r}=\frac{\Delta E}{2} \hat{\Sigma}_{z}^{(2)}+\hbar \omega_{0} \hat{a}^{\dagger} \hat{a}+U\left[\hat{\Sigma}_{+}^{(2)}+\hat{\Sigma}_{-}^{(2)}\right]\left(\hat{a}+\hat{a}^{\dagger}\right)^{m_{2}}
$$

\subsection{Localization issues}

The models under discussion are relatively complicated, and it is appropriate to make use of all tools available to gain understanding of how the models work. In the case of the coupled oscillator and Dicke system, there is an interesting and relevant limit that we need to consider. In the limit that the number of oscillator quanta is very large, and also that the number of excited states of the two-level system is also very large, then it is possible to develop analytic solutions. For simplicity, we focus on a version of the model with single phonon exchange $\left(m_{2}=1\right)$, in which case the limit of the coupled oscillator and Dicke model approaches

$$
\hat{H}_{r} \rightarrow \Delta E N+\hbar \omega_{0} n+g \Delta E\left(\hat{\delta}_{+}^{n}+\hat{\delta}_{-}^{n}\right)\left(\hat{\delta}_{+}^{N}+\hat{\delta}_{-}^{N}\right)
$$


This Hamiltonian has localized even eigenfunctions given by

$$
\begin{aligned}
& \Phi_{N_{0}, n_{0}}^{e v e n}(N, n)=
\end{aligned}
$$

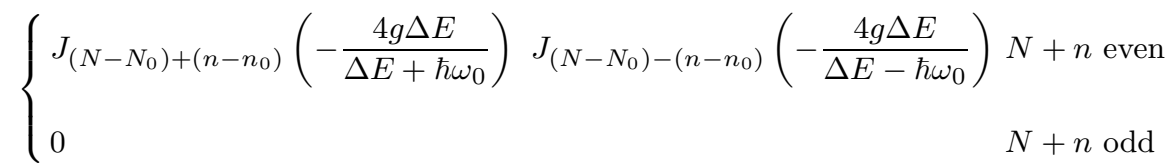

The associated eigenvalues are given by

$$
E_{N_{0}, n_{0}}=N_{0} \Delta E+n_{0} \hbar \omega_{0}
$$

Very similar eigenfunctions occur for $N_{0}+n_{0}$ odd.

\subsection{Dynamics and slowly varying $g$}

Although we have an important limit of the coupled model in which localized solutions occur. What this means specifically is that the system will develop fluctuations in $n$ and $N$ that are on the order of the coupling constant $g$. For net conversion of nuclear energy into phonon energy, a simple fluctuation is not helpful - we need large numbers of oscillator quanta to be converted, far more than $g$. Consequently, we need to understand what effects are capable of making the eigenfunctions delocalized. In addition, we might expect that the rate for net energy transfer between the two systems may be relatively small.

Since $g$ is essentially the interaction matrix element divided by the transition energy, it can be on the general order of the number of receiver-side two-level systems (if we are able to arrange for half to be excited), since the underlying interaction strength per nucleus is probably on the order of a few $\mathrm{MeV}$. Hence, the coupled system in this kind of model is very strongly coupled, and fluctuations will be very large. As a result, the coupling strength $g$ will vary over the eigenfunction in the $(N, n)$ configuration space, which can result in a finite (but small) rate of net energy transfer.

From the results of numerical calculations some intuition has emerged concerning the dynamics associated with this system (when $g$ is not fixed as a constant). This kind of model appears to be able to make rapid transitions between states with different $N$ (two-level system excitation) with little phonon exchange. Such dynamics can occur on a frequency scale that is on the general order of $g \Delta E / \hbar$. Transient solutions can be developed which show occupation of states that correspond to $\Delta E / \hbar \omega_{0}$ phonons exchanged on a frequency scale that is on the general order of

$g \omega_{0}$. However, these solutions tend to have roughly equal amounts of components with fewer or more phonons.

We are looking instead for slower dynamics where all states occupied have roughly the same energy, in which nuclear energy is traded off for oscillator energy. Since constant $g$ produces localization, one might expect that variations in 


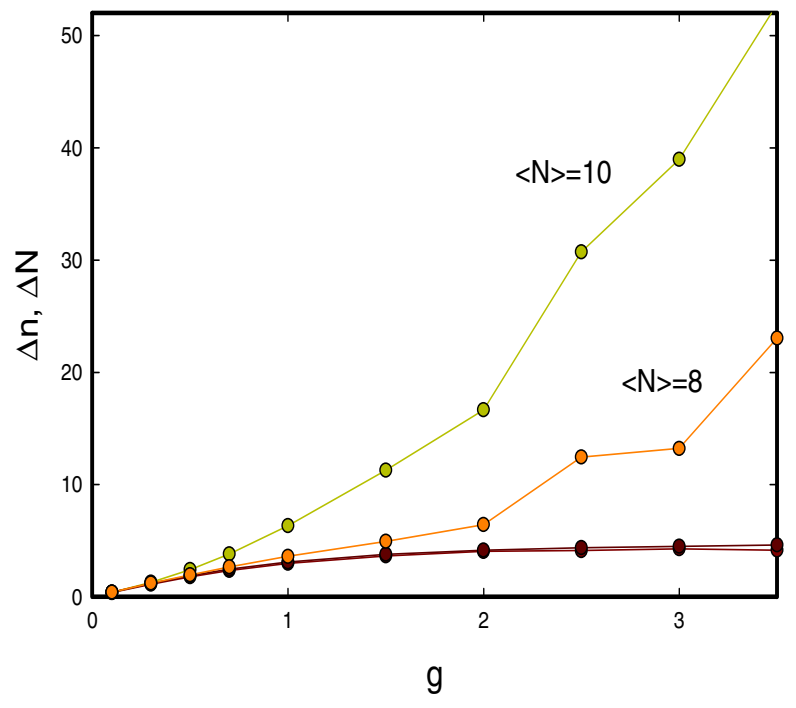

Figure 3. Spread in oscillator number $\Delta n$ (upper points) and in Dicke number $\Delta N$ (lower points) for eigenfunctions centered near $\langle N\rangle=8$ and $\langle N\rangle=10$. The calculations performed for a set of 15 two-level systems coupled to an oscillator with large $n$.

$g$ will be responsible for delocalization and consequently net energy exchange between the coupled systems. To study this we have in earlier work carried out a change of basis in which the $(N, n)$ configuration space is replaced by an expansion in the localized basis functions discussed above. The general conclusion is that under conditions where the approach is valid (slowly varying $g$ ), the system would be expected to develop localized solutions made up of superpositions of localized basis functions.

\section{Delocalization}

There are perhaps some positive things to be said about the problem of delocalization. Suppose one simply sets up the coupled Hamiltonian and begins solving for eigenfunctions, keeping track of how localized the eigenfunctions are as a function of model parameters. To simplify things, consider the limit where the number of oscillator quanta is sufficiently large that $g$ does not depend on $n$. If we focus on finite models, and look at the eigenfunctions that are roughly centered near the midrange of the problem in $n$ and in $N$, we find a number of interesting conclusions. At small $g$, we see localization, which can be measured quantitatively through the ratio of the spread in $n$ compared to the spread in $N$

$$
r=\frac{\Delta n}{\Delta N}
$$

In the case of localized solutions, this ratio is essentially unity. As $g$ increases, the eigenfunctions begin to approach the boundaries in $N$, which keeps them from expanding arbitrarily in this direction, and which also causes them to elongate in 
the $n$ direction (since the underlying symmetry that causes localization becomes broken). This effect is illustrated in Figure 3 in the case of 15 two-level systems.

To understand the associated dynamics we have done some computations in order to find out at what frequencies dynamical transitions occur. In the calculations so far there is a strong response (as measured through off-diagonal matrix elements $\left.\left\langle\Phi_{i}|n| \Phi_{j}\right\rangle\right)$ at

$$
\delta \epsilon=2 \hbar \omega_{0}
$$

There are also lesser contributions at both lower and higher frequencies. The model then has in it a characteristic time $\left(1 / 2 \omega_{0}\right)$ to transfer some unit of energy between the two systems. It remains to be determined whether this is a local oscillation, or whether it is characteristic of a more extended transfer process.

\section{Dynamics of coupled Dicke systems}

Based on the discussion above, we are motivated to consider a new kind of model to describe the dynamics of the $\mathrm{D}_{2}$ system and the coupled receiver system. If we assume that significant conversion of excitation on the receiver side to phonons is slow, then it seems productive to neglect it completely in order to develop a description of the excitation transfer dynamics associated with the populations.

There is the question of resonance. On the one hand, there is no reason to expect there to be receiver nuclei transitions that are matched to the energy difference between $\mathrm{D}_{2}$ and ${ }^{4} \mathrm{He}$. On the other hand, there is no excitation transfer between unmatched Dicke systems in the limit of hindered coupling and no phonon exchange. This sharply limits the possibilities. If the energy of the nuclear excitation is fixed and off of resonance, then the energy difference must be made up through phonon exchange in the strongly-coupled oscillator and Dicke system on the receiver side. Alternatively, it may be the case that the nuclear excitation energy on the receiver side is determined by the energy involved in the excitation transfer. The argument in support of this is that one would expect the nuclei to have a very broad energy response (perhaps roughly proportional to the giant dipole response, but not the same since our excitation is not dipolar) under the conditions of excitation transfer. After all, if a quantum of energy is transferred, and if the nuclear system does not loose the energy, then it should be available for transferring back. Overall in this scenario the transfer process would create a precise resonance.

From our perspective, either scenario is consistent in principle with the discussion which follows. There is a price to be paid, however, if the energy difference must be made up by phonon exchange. That price is a substantial reduction in efficiency, since it would be that the associated "oscillator strength" of the hybrid system must have a large energy spread.

\subsection{Evolution of resonant coupled Dicke systems}

Excitation transfer between resonant Dicke systems is governed by the Hamiltonian 


$$
\hat{H}=\frac{\Delta E}{2} \hat{\Sigma}_{z}^{(1)}+\frac{\Delta E}{2} \hat{\Sigma}_{z}^{(2)}+V e^{-G}\left[\hat{\Sigma}_{+}^{(1)} \hat{\Sigma}_{-}^{(2)}+\hat{\Sigma}_{-}^{(1)} \hat{\Sigma}_{+}^{(2)}\right]
$$

The development of evolution equations for expectation values of the pseudospin operators is highly nontrivial in the general case, and does not appear to lead to useful results. However, we have had success developing an approximate Ehrenfest calculation by adopting a restricted basis composed of degenerate states. The results of the Ehrenfest analysis, augmented empirically with loss, results in the following coupled equations

$$
\begin{array}{cl}
\frac{d}{d t} n_{1}(t)=v_{1}(t) & \frac{d}{d t} v_{1}(t)+\frac{v_{1}(t)}{T}=a(t) \\
\frac{d}{d t} n_{2}(t)+\frac{n_{2}(t)}{T}=v_{1}(t) & \frac{d}{d t} v_{2}(t)+\frac{v_{2}(t)}{T}=-a(t)
\end{array}
$$

where

$$
\begin{aligned}
a(t)=\frac{2 V^{2} e^{-2 G}}{\hbar^{2}}\left\{\left[N_{1}-n_{1}(t)\right][\right. & \left.N_{2}-n_{2}(t)\right]\left[n_{2}(t)-n_{1}(t)\right] \\
+ & n_{1}(t) n_{2}(t)\left[N_{1}-n_{1}(t)-N_{2}+n_{2}(t)\right] \\
& \left.+\left[N_{1}-n_{1}(t)\right] n_{2}(t)-\left[N_{2}-n_{2}(t)\right] n_{1}(t)\right\}
\end{aligned}
$$

Here $n_{1}(t)$ and $n_{2}(t)$ are the average number of excited states in system $1\left(\mathrm{D}_{2}\right.$ side $)$ and system 2 (receiver side), and $v_{1}(t)$ and $v_{2}(t)$ are the associated velocities. The acceleration is $a(t)$. The Dicke number for the two sides are $N_{1}$ and $N_{2}$, which can be as large as the total number of nuclei involved on each side.

\subsection{Example}

In Figure 4 we show results from a numerical calculation of the evolution equations for resonantly coupled Dicke systems. In this calculation we have assumed that there are ten times more receiver side nuclei in the ground state than there are $\mathrm{D}_{2}$ molecules in the upper state. In addition, we have set the relaxation time to be matched to the coherent transfer rate. If we use a much slower relaxation time, then we observe population returning from the receiver side. If we use a much faster relaxation time, then we observe a slower net transfer of excitation. The problem in this case is that if the receiver-side nuclei decay rapidly, then there are relatively few around to provide a (receiver-side) Dicke enhancement of the acceleration. In essence, the fastest net energy generation rate is obtained when we match the excitation transfer rate with the receiver-side loss. 


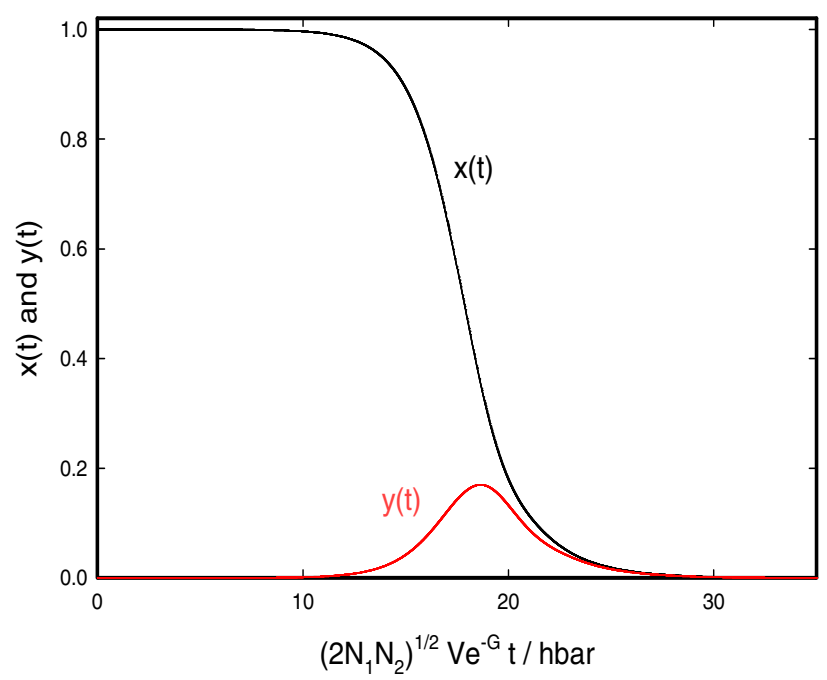

Figure 4. Calculation of normalized populations $x(t)=n_{1}(t) / N_{1}$ and $y(t)=n_{2}(t) / N_{1}$ as a function of normalized time for conditions in which $N_{2}=10 N_{1}$.

\section{Avoidance of loss}

We have noted previously in our quantum flow calculations that the probability amplitude tends to avoid regions of high loss. A very much simplified version of this argument can be given. Consider the situation of two states that are coupled, one loss free and the other with high loss:

$$
\begin{gathered}
i \hbar \frac{\partial}{\partial t} c_{0}(t)=H_{0} c_{0}(t)+V_{01} c_{1}(t) \\
i \hbar \frac{\partial}{\partial t} c_{1}(t)=\left[H_{1}-i \frac{\hbar \gamma_{1}}{2}\right] c_{1}(t)+V_{10} c_{0}(t)
\end{gathered}
$$

We initialize the system such that $c_{0}(0)=1$, and ask what happens later on. The exact solution is more complicated than what we are interested in here; however, a useful simplification is allowed if we assume that the level 1 loss is very strong. In this case, $c_{0}$ decays slowly as we will find, and we may assume that the $c_{1}(t)$ probability amplitude is determined in the steady state by

$$
c_{1}(t)=\left[E-H_{1}+i \hbar \gamma_{1} / 2\right]^{-1} V_{10} c_{0}(t)
$$

with $E$ approximately $H_{0}$. The resulting evolution of level 0 is then

$$
i \hbar \frac{\partial}{\partial t} c_{0}(t)=H_{0} c_{0}(t)+\frac{\left|V_{01}\right|^{2}}{E-H_{1}+i \hbar \gamma_{1} / 2} c_{1}(t)
$$

The effective loss seen by level 0 is then 


$$
\gamma=\frac{\left|V_{01}\right|^{2}}{\left(E-H_{1}\right)^{2}+\left(\hbar \gamma_{1} / 2\right)^{2}} \gamma_{1}
$$

Increasing the level 1 loss $\gamma_{1}$ to ever larger values has the perhaps unexpected effect of decreasing the level 0 loss. In essence, the probability amplitude tends to avoid level 1 as it gets increasingly lossy. Alternatively, one can view this result as indicated that the maximum loss from level 0 is obtained when the loss is matched to the coherent transition rate.

\section{Nuclear models}

We have made progress on the other half of the problem which involves the calculation of the nuclear response in the presence of phonon-mediated excitation transfer. The calculation of phonon exchange matrix elements requires the inclusion of the nuclear center of mass coordinates as phonon operators. Results relevant to such calculations for the four-body problem is discussed in another paper submitted to this proceedings. [6]

We have in addition developed a new strategy for the calculation of the nuclear response which may be worth discussing briefly. The lattice generalization of the resonating group method that we have developed allows us to include phonon exchange explicitly in calculating nuclear interaction matrix elements. It seems that the most relevant and perhaps cleanest calculation which is needed is a second-order process in which an initial nuclear system receives energy via excitation transfer in a first phonon mediated strong force interaction, then evolves as a daughter plus neutral, and after a while makes a transition to a final state nuclear system. The basic interaction matrix element can be represented as

$$
\begin{aligned}
& M_{f i}(E)= \\
& \sum_{i n t}\left\langle\Psi_{f}(\mathbf{q}) \Phi_{f}\left(\xi_{1}, \cdots, \xi_{A}\right)\left|V_{N}\right| \Phi_{i n t}\left[E-\hat{H}_{i n t}\right]^{-1}\left\langle\Phi_{i n t}\left|V_{N}\right| \Phi_{i}\left(\xi_{1}, \cdots, \xi_{A}\right) \Psi_{i}(\mathbf{q})\right\rangle\right\rangle
\end{aligned}
$$

In the initial state, we see an internal nuclear wavefunction $\Phi_{i}\left(\xi_{1}, \cdots, \xi_{A}\right)$, with $\xi_{j}$ as relative nucleon coordinates, embedded in a lattice (or condensed matter system) described by $\Psi_{i}(\mathbf{q})$. Transitions are mediated by the strong force, described here by the nuclear potential $V_{N}$. The intermediate internal nuclear wavefunctions for the different possible intermediate configurations are $\Phi_{i n t}$. The lattice wavefunction in the intermediate state is implied through the specific bra and ket arrangements, but since there is a neutral involved in the intermediate state, the phonon modes of the lattice wavefunction are rotated through a Duschinsky transform (leading to intermediate wavefunctions of the form $\Psi_{\text {int }}(\mathbf{A} \cdot \mathbf{q})$. Finally, the neutral and daughter come back together through the second potential interaction, producing final state nuclear $\Phi_{f}\left(\xi_{1}, \cdots, \xi_{A}\right)$ and lattice wavefunctions $\Psi_{f}(\mathbf{q})$. In this case, 


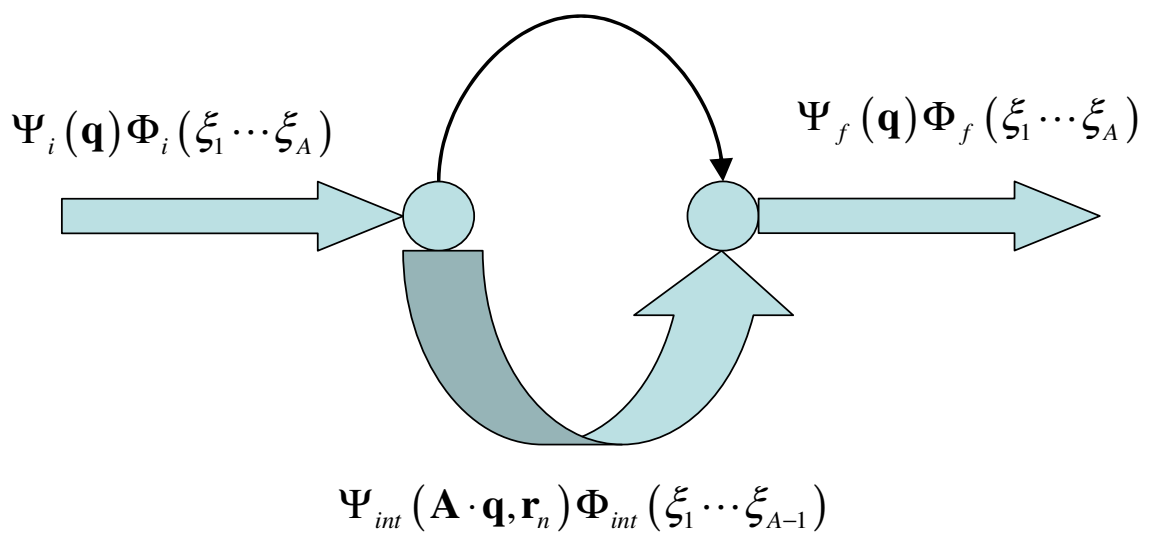

Figure 5. Feynman-type diagram for matrix element involving intermediate lattice plus neutral states.

the phonon basis for the initial and final state lattice wavefunctions are locally the same, since there is no net mass change. There could be rearrangements in either the initial or final state systems, such as would occur if $\mathrm{D}_{2}$ or $H T$ molecular systems are involved. However, when the nuclei are close enough to interact, the lattice will simply see four nucleons and two charges.

This basic calculation is involved both in phonon-mediated transitions of the form $\mathrm{D}_{2} \rightarrow \mathrm{n}+{ }^{3} \mathrm{He} \rightarrow{ }^{4} \mathrm{He}$ on the $\mathrm{D}_{2}$ side, as well as ${ }^{A} \mathrm{Z} \rightarrow \mathrm{n}^{m}+{ }^{A-m} \mathrm{Z} \rightarrow{ }^{A} \mathrm{Z}$ transitions on the receiver side. The matrix element can be represented by a Feynman-like diagram that is illustrated in Figure 5. Implicit in the expression for the matrix element and also in the diagram is the point of view that the lattice (or condensed matter system) separates from a neutral (in association with an excitation transfer event), evolves as a daughter lattice plus neutral in the intermediate state, and then comes back together to form the final state lattice. Instead of a vacuum language that focuses on nuclei and nucleons, in the formulation under discussion, the interaction is with a nuclei which is part of a lattice, and it is helpful to think of it as a neutral plus lattice separation. Such a view makes plain what the calculation involves at the outset, and makes clear that it is in fact a fundamentally different calculation than what is involved in related vacuum calculations.

\section{Summary}

We are moving toward viewing relevant models as involving the hindered coupling between a Dicke system for the $\mathrm{D}_{2} /{ }^{4} \mathrm{He}$ side and a hybrid quantum system on the receiver side composed of one or more Dicke systems strongly coupled to one or more highly excited oscillators. In previous work, we have been interested in the possibility that loss can break the symmetry of the coupled oscillator and Dicke Hamiltonian in order to allow the conversion of nuclear energy to phonon energy. 
Here we have shown that the boundaries associated with the Dicke system can accomplish the same basic function in a relevant strong coupling limit. Such a model is capable of transferring energy on a timescale relevant to the oscillator frequency, but we have not as yet clarified how much energy is transferred during that time (other than the fact that it can be at least as large as one nuclear quantum).

We presented the results of an Ehrenfest analysis of coupled resonant Dicke systems, augmented with loss to take into account energy coupling with the lattice in an empirical way. The resulting evolution equations should be relevant to the dynamics of fusion reactions under conditions where the $\mathrm{D}_{2}$ source is fixed (not replenished). Progress on the nuclear calculations was discussed briefly as well.

\section{References}

1. M. Fleischmann, S. Pons and M. Hawkins, J. Electroanal Chem., 201, p.301 (1989); Errata, 263 p. 187 (1990). See also M. Fleischmann, S. Pons, M.W. Anderson, L.J. Li and M. Hawkins, J. Electroanal. Chem., 287, p. 293 (1990).

2. S.E. Jones, E.P. Palmer, J.B. Czirr, D.L. Decker, G.L. Jensen, J.M. Thorne, S.F. Taylor and J. Rafelski, "Observation of cold nuclear fusion in condensed matter," Nature $\mathbf{3 3 8}$ 737 (1989).

3. P. L. Hagelstein, Proceedings of the Ninth International Conference on Cold Fusion, May 2002 Beijing, China, edited by X. Z. Li, p. 121.

4. P. L. Hagelstein Proceedings of the Tenth International Conference on Cold Fusion, Aug. 2003 Cambridge, MA, edited by P. L. Hagelstein and S. R. Chubb, p. 837.

5. P. L. Hagelstein Proceedings of the Eleventh International Conference on Cold Fusion, Nov. 2004 Marseilles, France, edited by J. P. Biberian, (in press).

6. I. Chaudhary and P. L. Hagelstein, this proceedings. 